\title{
STABILITY AND BIFURCATION ANALYSIS FOR A DELAYED SHIMIZU-MORIOKA MODEL
}

\author{
XIN ZHANG \\ Received 05 February, 2018
}

\begin{abstract}
This paper is concerned with a Shimizu-Morioka model with constant delays. Its stability of the equilibrium is investigated and the existence of Hopf bifurcations is demonstrated by analyzing the associated characteristic equation. Furthermore, the explicit formulae determining the stability and the direction of periodic solutions bifurcating from Hopf bifurcations are obtained by applying the center manifold theory and the normal form method. Finally, special attention is paid to numerical simulations in order to verify the theoretical predictions.
\end{abstract}

2010 Mathematics Subject Classification: 34C23; 35B10; 37C75; 37G05

Keywords: Shimizu-Morioka model, time delays, stability, local Hopf bifurcation

\section{INTRODUCTION}

The original Shimizu-Morioka model [16] is described by the following equation:

$$
\left\{\begin{array}{l}
\dot{x} \equiv \frac{\mathrm{d} x}{\mathrm{~d} t}=y, \\
\dot{y} \equiv \frac{\mathrm{d} y}{\mathrm{~d} t}=x-\beta y-x z, \\
\dot{z} \equiv \frac{\mathrm{d} z}{\mathrm{~d} t}=-\alpha z+x^{2},
\end{array}\right.
$$

where $(x, y, z) \in \mathbb{R}^{3}$ are the state variables and $\alpha, \beta$ are real positive parameters. This model has been proposed as a simplified and an alternative model for studying the dynamics of the well-known Lorenz system for large Rayleigh numbers (Ra), in which complex behavior of the trajectories has been discovered by means of computer simulation. As in the Lorenz model, the Shimizu-Morioka model is invariant with respect to the substitution $(x, y, z) \rightarrow(-x,-y, z)$. The model received much attention due to its stability to describe bifurcation of the associated Lorenz like strange attractors [15], e.g., taking $\alpha=0.45$ and $\beta=0.75$ (see Figure 1). In case that from the dynamical system of equation (1.1) follows that

$$
\nabla V=\frac{\partial X}{\partial x}+\frac{\partial Y}{\partial y}+\frac{\partial Z}{\partial z}=-(\alpha+\beta)<0,
$$

then the system is known to be a dissipative one. According to the definition of the dissipative system [19], a volume element $V_{0}$ is contracted by the flow into a volume 


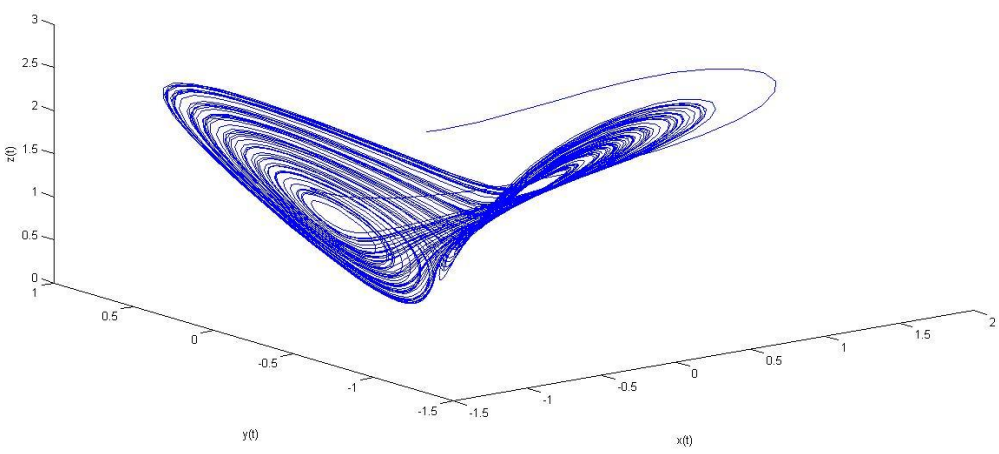

FIGURE 1. Phase portraits of Shimizu-Morioka model in the $x-$ $y-z$ space.

element $V_{0} e^{\nabla V_{t}}$ in time $t$. Hence, each volume containing the system orbit shrinks to zero as $t \rightarrow \infty$ at an exponential rate.

During the past decades, there are many researchers focus on the study of dynamical behaviour analysis of Shimizu-Morioka model. In particular, they use feedback control laws and delay feedback control method to study the local and global stabilization and bifurcation of Shimizu-Morioka model[9] [3][18].

Delay differential equations (DDEs) are well-known as differential equations with time delay term, which exhibit considerably more complex dynamical behavior than ordinary differential equations (ODEs) since the delays could cause a stable equilibrium to become unstable and fluctuate. The research of the dynamical behaviors for DDEs has received much attention in interdisciplinary subjects including natural science [11][2][7], engineering [1], life sciences [17] and others [20][21]. So, there's great significance to study the delayed Shimizu-Morioka model as follows:

$$
\left\{\begin{array}{l}
\dot{x}(t)=y(t), \\
\dot{y}(t)=x(t-\tau)-\beta y(t)-x(t-\tau) z(t), \\
\dot{z}(t)=-\alpha z(t)+x^{2}(t-\tau),
\end{array}\right.
$$

where $\tau$ is a non-negative constant delay term.

We define $\mathbb{R}_{+} \equiv\{x \in \mathbb{R}: x \geq 0\}$, int $\mathbb{R}_{+} \equiv\{x \in \mathbb{R}: x>0\}$. Denoted by $C\left([-\tau, 0], \mathbb{R}_{+}\right)$, the infinite dimensional Banach space of continuous functions from the interval $[-\tau, 0]$ into $\mathbb{R}_{+}$, equipped with the uniform norm. We assume initial data for model (1.3) is taken from $X=C\left([-\tau, 0], \mathbb{R}_{+}\right) \times C\left([-\tau, 0], \mathbb{R}_{+}\right) \times C\left([-\tau, 0], \mathbb{R}_{+}\right)$. The variables $x(t), y(t)$ and $z(t)$ in model (1.3) belong to $X$ for $t \in[-\tau, 0]$. 
In this paper, we will take the delay $\tau$ as bifurcation parameter and show that when $\tau$ passes through the critical values, the equilibrium loses its stability and the Hopf bifurcation occurs. The rest of this paper is organized as follows. In Sect.2, the stability and the existence of Hopf bifurcation parameter are determined. In Sect.3, we give details of the bifurcation values that describe the direction of the Hopf bifurcation and the stability for the bifurcated periodic solution using the center manifold theory and the normal form method introduced by Hassard et al. [5]. Finally, some numerical simulations and conclusions are given to illustrate theoretical predictions in Sect.4.

\section{THE EXISTENCE OF LOCAL HOPF BIFURCATION}

In this section, we firstly give out the existence and stability of the equilibrium for system (1.3). It has three equilibrium points $E(0,0,0), E_{+}(\sqrt{\alpha}, 0,1)$ and $E_{-}(-\sqrt{\alpha}, 0,1)$. Due to the symmetry of the system, it is sufficient to study the existence of local Hopf bifurcations occurring at $E_{+}$.

Let $x_{1}(t)=x(t)-\sqrt{\alpha}, x_{2}(t)=y(t), x_{3}(t)=z(t)-1$, then system (1.3) becomes

$$
\left\{\begin{array}{l}
\dot{x}_{1}(t)=x_{2}(t), \\
\dot{x_{2}}(t)=-\beta x_{2}(t)-\sqrt{\alpha} x_{3}(t)-x_{1}(t-\tau) x_{3}(t), \\
\dot{x_{3}}(t)=2 \sqrt{\alpha} x_{1}(t-\tau)-\alpha x_{3}(t)+x_{1}^{2}(t-\tau),
\end{array}\right.
$$

the linearization of system (2.1) is

$$
\left\{\begin{array}{l}
\dot{x}_{1}(t)=x_{2}(t), \\
\dot{x_{2}}(t)=-\beta x_{2}(t)-\sqrt{\alpha} x_{3}(t), \\
\dot{x_{3}}(t)=2 \sqrt{\alpha} x_{1}(t-\tau)-\alpha x_{3}(t) .
\end{array}\right.
$$

The associated characteristic equation of (2.2) is

$$
\left|\begin{array}{ccc}
\lambda & -1 & 0 \\
0 & \lambda+\beta & \sqrt{\alpha} \\
-2 \sqrt{\alpha} e^{-\lambda \tau} & 0 & \lambda+\alpha
\end{array}\right|=0,
$$

i.e.

$$
\lambda^{3}+(\alpha+\beta) \lambda^{2}+\alpha \beta \lambda+2 \alpha e^{-\lambda \tau}=0 .
$$

When $\tau=0$, Eq.(2.3) becomes

$$
\lambda^{3}+(\alpha+\beta) \lambda^{2}+\alpha \beta \lambda+2 \alpha=0 .
$$

We give out the assumption $\left(\mathrm{H}_{1}\right)(\alpha+\beta) \beta>2$.

By Routh-Hurwitz stability criterion, we obtain

Lemma 1. Assume that $\left(H_{1}\right)$ holds, then when $\tau=0$, the equilibrium $E_{+}$of system (2.1) is asymptotically stable. 
If $i \omega(\omega>0)$ is a root of Eq.(2.3), then

$$
-i \omega^{3}-(\alpha+\beta) \omega^{2}+i \omega \alpha \beta+2 \alpha(\cos \omega \tau-i \sin \omega \tau)=0 .
$$

Separating the real and imaginary parts, we obtain

$$
\left\{\begin{array}{l}
-(\alpha+\beta) \omega^{2}+2 \alpha \cos \omega \tau=0, \\
\alpha \beta \omega-\omega^{3}-2 \alpha \sin \omega \tau,
\end{array}\right.
$$

it follows from (2.6) that

$$
\left\{\begin{array}{l}
\sin (\omega \tau)=\frac{\omega\left(\alpha \beta-\omega^{2}\right)}{2 \alpha}, \\
\cos (\omega \tau)=\frac{(\alpha+\beta) \omega^{2}}{2 \alpha} .
\end{array}\right.
$$

By $\sin ^{2}(\omega \tau)+\cos ^{2}(\omega \tau)=1$, then (2.3) is equivalent to

$$
\omega^{6}+\left(\alpha^{2}+\beta^{2}\right) \omega^{4}+\alpha^{2} \beta^{2} \omega^{2}-4 \alpha^{2}=0 .
$$

Let $z=\omega^{2}$, Eq.(2.8) becomes

$$
z^{3}+\left(\alpha^{2}+\beta^{2}\right) z^{2}+\alpha^{2} \beta^{2} z-4 \alpha^{2}=0 .
$$

From (2.8), we have $h(z)=z^{3}+\left(\alpha^{2}+\beta^{2}\right) z^{2}+\alpha^{2} \beta^{2} z-4 \alpha^{2}$. Then, $h^{\prime}(z)=3 z^{2}+$ $2\left(\alpha^{2}+\beta^{2}\right) z+\alpha^{2} \beta^{2}$. Denote $\Delta=\left(\alpha^{2}+\beta^{2}\right)^{2}-3 \alpha^{2} \beta^{2}$, obviously $\Delta \geq \alpha^{2} \beta^{2}>0$, the equation $3 z^{2}+2\left(\alpha^{2}+\beta^{2}\right) z+\alpha^{2} \beta^{2}=0$ has two real negative roots:

$$
z_{1}^{*}=\frac{-\left(\alpha^{2}+\beta^{2}\right)+\sqrt{\Delta}}{3}, z_{2}^{*}=\frac{-\left(\alpha^{2}+\beta^{2}\right)-\sqrt{\Delta}}{3} .
$$

Noticing that $h(0)<0, \lim _{z \rightarrow+\infty} h(z)=+\infty$. Eq.(2.9) has one positive real roots $z$, and Eq.(2.8) has two real root $\omega_{k}(k=1,2)$, suppose $\omega_{1}=-\sqrt{z}, \omega_{2}=\sqrt{z}$. Substituting $\omega_{k}(k=1,2)$ into $(2.7)$, we have

$$
\tau_{k}^{j}=\frac{1}{\omega_{k}}\left\{\arccos \left(\frac{(\alpha+\beta) \omega_{k}^{2}}{2 \alpha}\right)+2 j \pi\right\},
$$

where $k=1,2$ and $j=0,1,2, \ldots$, then $i \omega_{k}(k=1,2)$ is a pair of pure imaginary roots of Eq.(2.3) with $\tau_{k}^{j}$. Define

$$
\tau_{0}=\tau_{k}^{(0)}=\min _{k \in\{1,2\}}\left\{\tau_{k}^{(0)}\right\}
$$

Lemma 2. When $\tau=\tau_{k}^{j}(k=1,2$ and $j=0,1,2, \ldots)$, then (2.3) has a pair of pure imaginary roots $i \omega_{k}$, and all other roots of (2.3) have nonzero real parts.

Let $\lambda(\tau)=\alpha(\tau) \pm i \omega(\tau)$ be the roots of (2.3) near $\tau=\tau^{j}$ satisfying $\alpha\left(\tau^{j}\right)=$ $0, \omega\left(\tau^{j}\right)=\omega_{k}(j=0,1,2, \cdots)$. By the theory of functional differential equation [5], for $\forall \tau^{j}, \exists \varepsilon>0$ s.t. $\lambda(\tau)$ in $\left|\tau-\tau^{j}\right|<\varepsilon$ about $\tau$ is continuous and differentiable. 
Lemma 3. The transversality condition

$$
\left.\left[\frac{d(\operatorname{Re} \lambda(\tau))}{d \tau}\right]\right|_{\tau=\tau_{j}}>0 .
$$

Proof. Substituting $\lambda(\tau)$ into Eq.(2.3) and taking the derivative with respect to $\tau$, we have

by (2.7), we get

$$
\left(\frac{d \lambda(\tau)}{d \tau}\right)^{-1}=\frac{\left[3 \lambda^{2}+2(\alpha+\beta) \lambda+\alpha \beta\right] e^{\lambda \tau}}{2 \alpha \lambda}-\frac{\tau}{\lambda},
$$

$$
\begin{aligned}
{\left.\left[\frac{d(\operatorname{Re} \lambda(\tau))}{d \tau}\right]\right|_{\tau=\tau_{j}} } & =\operatorname{Re}\left[\frac{\left[3 \lambda^{2}+2(\alpha+\beta) \lambda+\alpha \beta\right] e^{\lambda \tau}}{2 \alpha \lambda}\right]_{\tau=\tau_{j}} \\
& =\operatorname{Re}\left[\frac{\left[-3 \omega^{2}+2 i(\alpha+\beta) \omega+\alpha \beta\right]\left(\cos \omega_{k} \tau_{k}^{j}+i \sin \omega_{k} \tau_{k}^{j}\right)}{2 i \alpha \omega}\right]_{\tau=\tau_{j}} \\
& =\frac{\alpha^{2} \beta^{2}+3 \omega^{4}+2\left(\alpha^{2}+\beta^{2}\right) \omega^{2}}{4 \alpha^{2}}>0 .
\end{aligned}
$$

By above Lemmas and the conditions for Hopf bifurcation theorem [6], we have the following result:

Theorem 1. Assume that $\left(H_{1}\right)$ holds.

$$
\tau_{k}^{j}=\frac{1}{\omega_{k}}\left\{\arccos \left(\frac{(\alpha+\beta) \omega_{k}^{2}}{2 \alpha}\right)+2 j \pi\right\}
$$

are Hopf bifurcation values at $E_{+}$, where $i \omega_{k}(k=1,2)$ are the roots of (2.3). And $E_{+}$is locally asymptotically stable for $\tau \in\left[0, \tau_{1}^{0}\right)$ and unstable where $\tau>\tau_{1}^{0}$.

\section{THE PROPERTY OF LOCAL HOPF BIFURCATION}

In the previous part, it is shown that system (2.1) undergoes Hopf bifurcation under certain conditions. In the following part, we will derive explicit formulae determining the direction of Hopf bifurcation and the stability of the periodic solutions bifurcating from $E_{+}$at $\tau^{j}(j=0,1,2 \cdots)$, by employing center manifold theory and normal form method. For convenience, denote $\tau^{j}$ by $\widetilde{\tau}$ and $\tau=\widetilde{\tau}+\mu, \mu \in R$, then $\mu=0$ is the Hopf bifurcation value for system (2.1).

The discussion will be divided into five steps.

Step 1. Transform system (2.1) into the abstract ODE.

The system (2.1) can locally be represented as the following DDE in $C=C\left([-1,0], \mathbb{R}^{3}\right)$

$$
\dot{u}(t)=L_{\mu}\left(u_{t}\right)+F\left(\mu, u_{t}\right),
$$


where $u(t)=\left(u_{1}(t), u_{2}(t), u_{3}(t)\right)^{T}, u_{t}(\theta)=u(t+\theta), L_{\mu}: C \rightarrow \mathbb{R}$ is a bounded linear operator and $F: \mathbb{R} \times C \rightarrow \mathbb{R}$ is continuous and differentiable with

$$
L_{\mu} \phi=(\tau+\mu)\left(\begin{array}{ccc}
0 & 1 & 0 \\
0 & -\beta & -\sqrt{\alpha} \\
0 & 0 & -\alpha
\end{array}\right)\left(\begin{array}{l}
\phi_{1}(0) \\
\phi_{2}(0) \\
\phi_{3}(0)
\end{array}\right)+\left(\begin{array}{ccc}
0 & 0 & 0 \\
0 & 0 & 0 \\
2 \sqrt{\alpha} & 0 & 0
\end{array}\right)\left(\begin{array}{l}
\phi_{1}(-1) \\
\phi_{2}(-1) \\
\phi_{3}(-1)
\end{array}\right)
$$

and

$$
F(\mu, \phi)=(\tau+\mu)\left(\begin{array}{c}
0 \\
-\phi_{1}(-1) \phi_{3}(0) \\
\phi_{1}^{2}(-1)
\end{array}\right),
$$

where $\phi=\left(\phi_{1}(\theta), \phi_{2}(\theta), \phi_{3}(\theta)\right) \in C$.

By the Riese representation theorem, there exists a $3 \times 3$ matrix whose elements are bounded variation function $\eta(\theta, \mu)$ in $\theta \in[-1,0]$ such that

$$
L_{\mu} \phi=\int_{-1}^{0} d \eta(\theta, \mu) \phi(\theta), \phi \in C,
$$

where $\eta(\theta, \mu)$ can be chosen as

$$
\eta(\theta, \mu)=(\tau+\mu)\left(\begin{array}{ccc}
0 & 1 & 0 \\
0 & -\beta & -\sqrt{\alpha} \\
0 & 0 & -\alpha
\end{array}\right) \delta(\theta)+\left(\begin{array}{ccc}
0 & 0 & 0 \\
0 & 0 & 0 \\
2 \sqrt{\alpha} & 0 & 0
\end{array}\right) \delta(\theta+1),
$$

where $\delta(\theta)$ is a Dirac delta function [12] and $\theta \in[-1,0]$.

For $\phi \in C$, let

$$
\begin{aligned}
& A(\mu) \phi(\theta)= \begin{cases}d \phi(\theta) / d \theta, & \theta \in[-1,0), \\
\int_{-1}^{0} d \eta(\mu, \theta) \phi(\theta), & \theta=0,\end{cases} \\
& R(\mu) \phi(\theta)= \begin{cases}0, & \theta \in[-1,0), \\
F(\mu, \phi), & \theta=0,\end{cases}
\end{aligned}
$$

then system (3.1) is equivalent to the following abstract operator equation

$$
\dot{u}(t)=A(\mu) u_{t}+R(\mu) u_{t} .
$$

Step 2. Calculate the eigenfunctions of $A=A(0)$ and the adjoint operator $A^{*}$ corresponding to $i \omega_{0} \widetilde{\tau}$ and $-i \omega_{0} \widetilde{\tau}$.

For $\psi \in C\left([0,1],\left(C^{3}\right)^{*}\right)$, where $\left(C^{3}\right)^{*}$ is the 3 -dimensional complex space of row vectors, we define the adjoint operator $A^{*}$ of $A$

$$
A^{*} \psi(s)= \begin{cases}-d \psi(s) / d s, & s \in(0,1] \\ \int_{-1}^{0} d \eta^{T}(\mu, t) \psi(-t), & s=0\end{cases}
$$


and the bilinear form is given by

$$
\langle\psi(s), \phi(\theta)\rangle=\bar{\psi}(0) \phi(0)-\int_{-\tau}^{0} \int_{\xi=0}^{\theta} \bar{\psi}^{T}(\xi-\theta) d \eta(\theta) \phi(\xi) d \xi,
$$

where $\eta(\theta)=\eta(\theta, 0)$. Then $A=A(0)$ and $A^{*}(0)$ are adjoint operators.

By [8], $\pm i \omega_{0} \widetilde{\tau}$ are eigenvalues of $A(0)$, so they are also eigenvalues of $A^{*}(0)$. Suppose that $q(\theta)=(1, \widetilde{\alpha}, \widetilde{\beta})^{T} e^{i \omega_{0} \theta}$ is the eigenfunction of $A(0)$ corresponding to the eigenvalue $i \omega_{0} \widetilde{\tau}$ and $q^{*}(s)=G\left(1, \widetilde{\alpha}^{*}, \widetilde{\beta}^{*}\right) e^{i \omega_{0} s}$ is the eigenfunction of $A^{*}$ corresponding to the eigenvalue $-i \omega_{0} \widetilde{\tau}$, where

$$
\begin{aligned}
& \widetilde{\alpha}=i \omega_{k}, \widetilde{\beta}=\frac{2 \sqrt{\alpha} e^{i \omega \tau}}{i \omega_{k}+\alpha}, \widetilde{\alpha}^{*}=\frac{1}{\beta-i \omega_{k}}, \widetilde{\beta}^{*}=\frac{\sqrt{\alpha}}{\left(\omega_{k}^{2}-\alpha \beta\right)+i \omega_{k}(\alpha+\beta)}, \\
& G=\left(1+\widetilde{\alpha}^{*}+\widetilde{\beta} \widetilde{\widetilde{\beta}}^{*}+\tau_{k} \widetilde{\alpha}^{*} \widetilde{\alpha}^{*} e^{-i \omega \tau}\right)^{-1},
\end{aligned}
$$

which assures that $\left\langle q^{*}(s), q(\theta)\right\rangle=1,\left\langle q^{*}(s), \bar{q}(\theta)\right\rangle=0$.

\section{Step 3. Obtain the reduced system on the center manifold.}

In this part, we will use the same notations as in [6] and compute the coordinates to describe the center manifold $C_{0}$ at $\mu=0$ (A local center manifold is in general not unique). Let $u_{t} \in C$ be the solution of system (3.2) when $\mu=0$, and define

$$
z(t)=\left\langle q^{*}, u_{t}\right\rangle, W(t, \theta)=u_{t}(\theta)-z(t) q(\theta)-\bar{z}(t) \bar{q}(\theta),
$$

where $z$ and $\bar{z}$ are local coordinates for center manifold $C_{0}$ in the direction of $q^{*}$ and $\bar{q}^{*}$. On the center manifold $C_{0}$, we have $W(t, \theta)=W(z(t), \bar{z}(t), \theta)$, where

$$
W(z, \bar{z}, \theta)=W_{20}(\theta) z^{2} / 2+W_{11}(\theta) z \bar{z}+W_{02}(\theta) \bar{z}^{2} / 2+\cdots .
$$

The existence of center manifold enables us to reduce (3.2) to an ODE on $C_{0}$. Note that $W$ is real if $u_{t}$ is real, we consider only real solutions. For solution $u_{t} \in C_{0}$ of system (3.2) at $\mu=0$,

$$
\begin{aligned}
\dot{z}(t) & =\left\langle q^{*}, \dot{u}_{t}\right\rangle=\left\langle q^{*}, A\left(u_{t}\right)+R\left(u_{t}\right)\right\rangle=\left\langle A^{*}\left(q^{*}\right), u_{t}\right\rangle+\left\langle q^{*}, R\left(u_{t}\right)\right\rangle \\
& =i \omega_{0} \widetilde{\tau} z(t)+\bar{q}^{*}(0) \cdot f(0, W(z, \bar{z}, \theta)+2 \operatorname{Re}\{z(t) q(\theta)\}), \\
& =i \omega_{0} \widetilde{\tau} z(t)+\bar{q}^{*}(0) \cdot f\left(0, u_{t}\right),
\end{aligned}
$$

with

$$
\bar{q}^{*}(0) \cdot f\left(0, u_{t}\right) \triangleq g(z, \bar{z}) .
$$

Rewrite (3.5), we obtain the reduced system on $C_{0}$ is described by

$$
\dot{z}(t)=i \omega_{0} \widetilde{\tau} z(t)+g(z, \bar{z}) \text {, }
$$

where

$$
g(z, \bar{z})=g_{20}(\theta) z^{2} / 2+g_{11}(\theta) z \bar{z}+g_{02}(\theta) \bar{z}^{2} / 2+g_{21}(\theta) z^{2} \bar{z} / 2+\cdots .
$$


We will mainly discuss Eq. (3.6) in the following part.

Step 4. Get the value of $g_{20}, g_{11}, g_{02}, g_{21}$ in (3.7).

In this part, we will calculate the coefficient $W_{20}(\theta), W_{11}(\theta), W_{02}(\theta), \cdots$, then substitute them in (3.5), and get the reduced system (3.6) on $C_{0}$.

It follows from (3.3) that

$$
\begin{aligned}
u_{t}(\theta)= & u(t+\theta)=W(t, \theta)+2 \operatorname{Re}\{z(t), q(\theta)\} \\
= & W_{20}(\theta) z^{2} / 2+W_{11}(\theta) z \bar{z}+W_{02}(\theta) \bar{z}^{2} / 2+(1, \widetilde{\alpha})^{T} e^{i \omega_{0} \widetilde{\tau} \theta} z \\
& +(1, \overline{\widetilde{\alpha}})^{T} e^{-i \omega_{0} \widetilde{\tau} \theta} \bar{z}+\cdots .
\end{aligned}
$$

We obtain

$$
\begin{aligned}
\phi_{3}(0) & =z \widetilde{\beta}+\overline{\bar{z}} \bar{\beta}+W^{(3)}(0)+\cdots, \\
\phi_{1}(-1) & =z e^{-i \omega_{0} \theta}+\bar{z} e^{i \omega_{0} \theta}+W^{(1)}(-1)+\cdots,
\end{aligned}
$$

It follows together with $F(\mu, \phi)$, we get

$$
f\left(0, u_{t}\right)=\widetilde{\tau}\left(\begin{array}{c}
0 \\
-\phi_{1}(-1) \phi_{3}(0) \\
\phi_{1}^{2}(-1)
\end{array}\right) .
$$

Substituting (3.8) into (3.9) (3.5) and comparing the coefficients with (3.7), we obtain

$$
\begin{aligned}
& g_{20}=2 \bar{D} \tau_{k}\left(-\widetilde{\alpha}^{*} \widetilde{\beta} e^{-i \omega_{0} \theta}+\widetilde{\beta}^{*} e^{-2 i \omega_{0} \theta}\right), \\
& g_{11}=\bar{D} \tau_{k}\left[-\widetilde{\alpha}^{*}\left(\widetilde{\beta} e^{i \omega_{0} \theta}+\widetilde{\beta} e^{-i \omega_{0} \theta}\right)+2 \widetilde{\beta}^{*}\right], \\
& g_{02}=2 \bar{D} \tau_{k}\left(-\widetilde{\alpha}^{*} \widetilde{\beta} e^{i \omega_{0} \theta}+\widetilde{\beta}^{*} e^{2 i \omega_{0} \theta}\right), \\
& g_{21}=2 \bar{D} \tau_{k}\left\{-\widetilde{\alpha}^{*} e^{-i \omega_{0} \theta} W_{11}^{(3)}(0)+\widetilde{\beta}^{*}\left[2 e^{-i \omega_{0} \theta} W_{20}^{(1)}(-1)\right]\right\} .
\end{aligned}
$$

Since there are $W_{20}(\theta)$ and $W_{11}(\theta)$ in $g_{21}$, we still need to compute them.

From (3.2) and (3.3), we have

$$
\dot{W}=\dot{u}_{t}-\dot{z} q-\dot{\bar{z}} \bar{q}= \begin{cases}A W-2 \operatorname{Re}\{g q(\theta)\}, & \theta \in[-1,0), \\ A W-2 \operatorname{Re}\{g q(0)\}+f_{0}, & \theta=0,\end{cases}
$$

where

$$
f_{0}=f_{z^{2}} z^{2} / 2+f_{z \bar{z}} z \bar{z}+f_{\bar{z}^{2}} \bar{z}^{2} / 2+f_{z^{2} \bar{z}} z^{2} \bar{z} / 2 \cdots .
$$

On the other hand, near the origin, on the center manifold $C_{0}$, according to (3.4) we obtain

$$
\begin{aligned}
\dot{W}= & W_{z} \dot{z}+W_{\bar{z}} \dot{\bar{z}}=\left[W_{20}(\theta) z+W_{11}(\theta) \bar{z}\right] \dot{z}+\left[W_{11}(\theta) z+W_{02}(\theta) \bar{z}\right] \dot{\bar{z}} \\
= & {\left[W_{20}(\theta) z+W_{11}(\theta) \bar{z}\right]\left(i \omega_{0} z+g(z, \bar{z})\right) } \\
& +\left[W_{11}(\theta) z+W_{02}(\theta) \bar{z}\right]\left(\bar{g}(z, \bar{z})-i \omega_{0} \bar{z}\right)+\cdots .
\end{aligned}
$$


Substituting (3.4) into right side of (3.11), equating terms of $\frac{z^{2}}{2}$ and $z \bar{z}$ of (3.11) with (3.12), we obtain

$$
\begin{aligned}
\left(2 i \omega_{0} I-A\right) W_{20}(\theta) & = \begin{cases}-g_{20} q(\theta)-\bar{g}_{02} \bar{q}(\theta), & \theta \in[-\widetilde{\tau}, 0), \\
-g_{20} q(0)-\bar{g}_{02} \bar{q}(0)+f_{z^{2}}, & \theta=0,\end{cases} \\
-A W_{11}(\theta) & = \begin{cases}-g_{11} q(\theta)-\bar{g}_{11} \bar{q}(\theta), & \theta \in[-\widetilde{\tau}, 0), \\
-g_{11} q(0)-\bar{g}_{11} \bar{q}(0)+f_{z \bar{z}}, & \theta=0 .\end{cases}
\end{aligned}
$$

According to the definition of $A$ and from (3.13)(3.14) for $\theta \in[-\widetilde{\tau}, 0)$, we get

$$
\begin{aligned}
& \dot{W}_{20}(\theta)=2 i \omega_{0} W_{20}(\theta)+g_{20} q(\theta)+\bar{g}_{02} \bar{q}(\theta), \\
& \dot{W}_{11}(\theta)=g_{11} q(\theta)+\bar{g}_{11} \bar{q}(\theta),
\end{aligned}
$$

solving for $W_{20}(\theta)$ and $W_{11}(\theta)$, we obtain

$$
\begin{aligned}
& W_{20}(\theta)=i g_{20} / \omega_{0} \cdot q(0) e^{i \omega_{0} \theta}+i \bar{g}_{02} / 3 \omega_{0} \cdot \bar{q}(0) e^{-i \omega_{0} \theta}+E_{1} e^{2 i \omega_{0} \theta}, \\
& W_{20}(\theta)=-i g_{11} / \omega_{0} \cdot q(0) e^{i \omega_{0} \theta}+i \bar{g}_{11} / \omega_{0} \cdot \bar{q}(0) e^{-i \omega_{0} \theta}+E_{2},
\end{aligned}
$$

where $E_{1}=\left(E_{1}^{(1)}, E_{1}^{(2)}, E_{1}^{(3)}\right)^{T} \in \mathbb{R}^{3}$ and $E_{2}=\left(E_{2}^{(1)}, E_{2}^{(2)}, E_{2}^{(3)}\right)^{T} \in \mathbb{R}^{3}$ are constant vectors.

In what follows we shall seek appropriate $E_{1}$ and $E_{2}$ in (3.15) and (3.16), respectively. According to the definition of $A$ and (3.13)(3.14) for $\theta=0$, we have

$$
\begin{aligned}
& \int_{-\widetilde{\tau}}^{0} d \eta(\theta) W_{20}(\theta)=2 i \omega_{0} W_{20}(0)+g_{20} q(0)+\bar{g}_{02} \bar{q}(0)-f_{z^{2}}, \\
& \int_{-\widetilde{\tau}}^{0} d \eta(\theta) W_{11}(\theta)=g_{11} q(0)+\bar{g}_{11} \bar{q}(0)-f_{z \bar{z}},
\end{aligned}
$$

where $\eta(\theta)=\eta(0, \theta)$ and

$$
f_{z^{2}}=\left(\begin{array}{c}
0 \\
\widetilde{\beta} e^{-i \omega_{0} \theta} \\
e^{-2 i \omega_{0} \theta}
\end{array}\right), f_{z \bar{z}}=\left(\begin{array}{c}
0 \\
\widetilde{\beta} e^{i \omega_{0} \theta}+\overline{\widetilde{\beta}} e^{-i \omega_{0} \theta} \\
2
\end{array}\right) .
$$

Substituting (3.15) into (3.17), we obtain

$$
\left(2 i \omega_{0} I-\int_{-\tau}^{0} e^{2 i \omega_{0} \theta} d \eta(\theta)\right) E_{1}=f_{z^{2}}
$$

that is

$$
\left(\begin{array}{ccc}
2 i \omega_{0} & 1 & 0 \\
0 & 2 i \omega_{0} & \sqrt{\alpha} \\
-2 \sqrt{\alpha} e^{-i \omega_{0} \tau} & 0 & 2 i \omega_{0}+\alpha
\end{array}\right) E_{1}=f_{z^{2}} .
$$


Similarly, substituting (3.16) into (3.18), we get

$$
\int_{-\widetilde{\tau}}^{0} d \eta(\theta) E_{2}=f_{z \bar{z}}
$$

that is

$$
\left(\begin{array}{ccc}
0 & 1 & 0 \\
0 & 0 & \sqrt{\alpha} \\
2 \sqrt{\alpha} e^{-i \omega_{0} \tau} & 0 & \alpha
\end{array}\right) E_{2}=f_{z \bar{z}} .
$$

We have got the value of $E_{1}$ and $E_{2}$ as (3.19) and (3.20) shown and the reduced system (3.6) finally.

Step 5. Obtain the key values $\mu_{2}, \beta_{2}, T_{2}$ to judge the property of the Hopf bifurcation.

Similar to calculate the Hopf bifurcation parameter of the ODE and as in [6], according to the analysis above and the expressions of $g_{20}, g_{11}, g_{02}$ and $g_{21}$, we can compute the following values:

$$
\begin{aligned}
c_{1}(0) & =i / 2 \omega_{0} \widetilde{\tau}\left(g_{11} g_{20}-2\left|g_{11}\right|^{2}-\left|g_{02}\right|^{2} / 3\right)+g_{21} / 2, \\
\mu_{2} & =-\operatorname{Re}\left\{c_{1}(0)\right\} / \alpha^{\prime}(\widetilde{\tau}), \\
\beta_{2} & =2 \operatorname{Re}\left\{c_{1}(0)\right\}, \\
T_{2} & =-\left[\operatorname{Im}\left\{c_{1}(0)\right\}+\mu_{2} \omega^{\prime}(\widetilde{\tau})\right] / \omega_{0},
\end{aligned}
$$

where $\lambda(\tau)=\alpha(\tau) \pm i \omega(\tau)$ is the characteristic root of (2.3), which is a continuous differentiable family. $\alpha^{\prime}(\widetilde{\tau})$ and $\omega^{\prime}(\widetilde{\tau})$ can be obtained by taking the derivation of two sides of (2.3) and taking values at $\widetilde{\tau}$.

These formulae give a description of the Hopf bifurcation periodic solution of system (1.1) at $\tau=\tau^{j}(j=0,1,2 \cdots)$ on the center manifold. Thus,we can obtain the following results according to the discussion about properties of Hopf bifurcating periodic solutions of dynamical system in [6]:

Theorem 2. For system (1.3), we assume that the conditions $\left(H_{1}\right)$ holds for the parameters.

(i) If $\mu_{2}>0\left(\mu_{2}<0\right)$, then the Hopf bifurcation is supercritical (subcritical);

(ii) If $\beta_{2}<0\left(\beta_{2}>0\right)$, then bifurcating periodic solution is stable (unstable);

(iii) If $T_{2}>0\left(T_{2}<0\right)$, then periods of periodic solutions increase (decrease).

\section{NUMERICAL SIMULATIONS AND CONCLUSIONS}

In this section, we shall give some numerical simulations to support the theoretical analysis discussed in previous section. In addition, we will give out our actual conclusions and the shortcomings. 
Firstly, we study the following specific model

$$
\left\{\begin{array}{l}
\dot{x}(t)=y(t), \\
\dot{y}(t)=x(t-\tau)-y(t)-x(t-\tau) z(t), \\
\dot{z}(t)=-2 z(t)+x^{2}(t-\tau),
\end{array}\right.
$$

with initial value is $(x(t), y(t), z(t))=(0.1,0.1,0.1)$ which satisfies $\left(\mathrm{H}_{1}\right)$. By computing, $E_{+}=(1.414,0,1)$ and $h(z)=z^{3}+5 z^{2}+4 z-16$ has only one positive root $z \approx 1.307$, get $\omega_{1} \approx-1.143, \omega_{2} \approx 1.143, \tau^{0} \approx 0.174$. By Theorem 1 , we get that $E_{+}$is asymptotically stable when $0 \leq \tau<\tau^{0}$, as Figure 2 illustrated, and $E_{+}$is unstable when $\tau>\tau^{0}$, as shown in Figure 3. Figure 2-3 are drawn with dde23 [14] which is a MatLab [10] package that integrates DDEs.
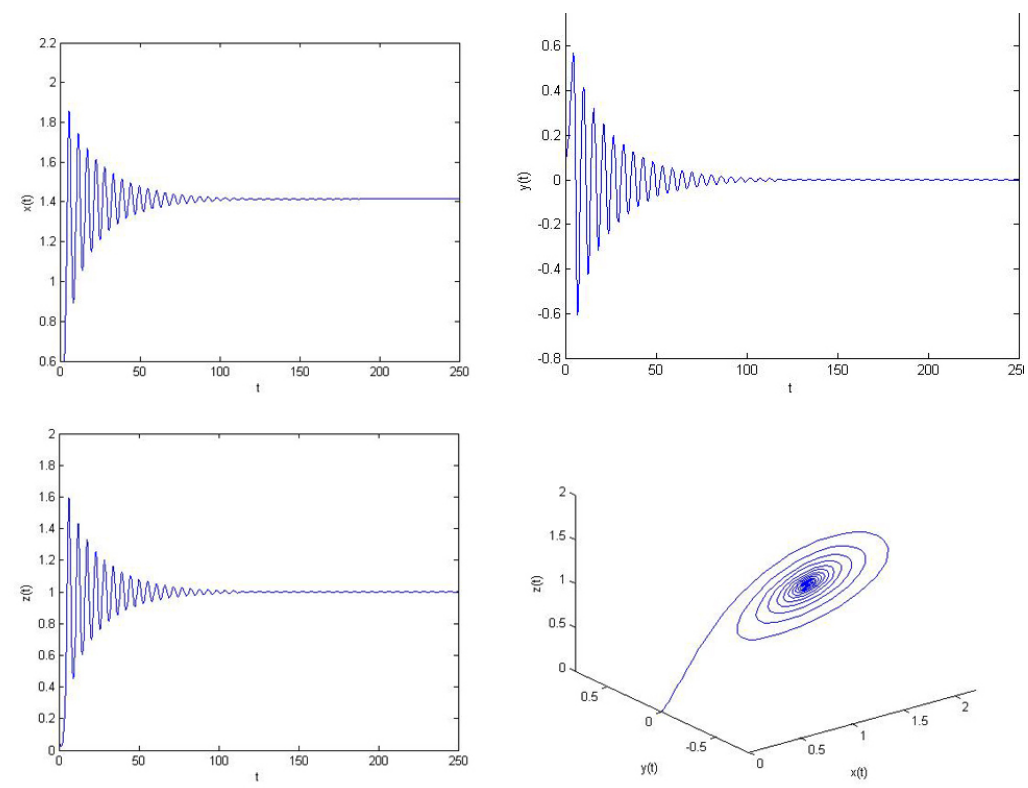

FIGURE 2. The equilibrium $E_{+}=(1.414,0,1)$ is locally asymptotically for system (2.1) with $\tau=0.1<\tau_{0}$.

In addition, the bifurcation diagram is shown by Figure 4, it is drawn using DDEBifTool [4][13] which is also a MatLab software package. The command window shows the equilibrium is $E_{0}=(1.4140,0,1.0000)$, Hopf Point 1 is at $\tau=0.1744$ with $\omega=1.1432$, Hopf Point 2 is at $\tau=5.6705$ with $\omega=1.1432$, Hopf Point 3 is at $\tau=11.1667$ with $\omega=1.1432$.

We take the $\tau-x$ space as example to explain the bifurcation meaning. At $\tau=$ $\tau^{0}=0.1744$ (the position of (a) dashed vertical line), the stable equilibrium point loses stability and becomes unstable, Hopf bifurcation occurs and generated the 

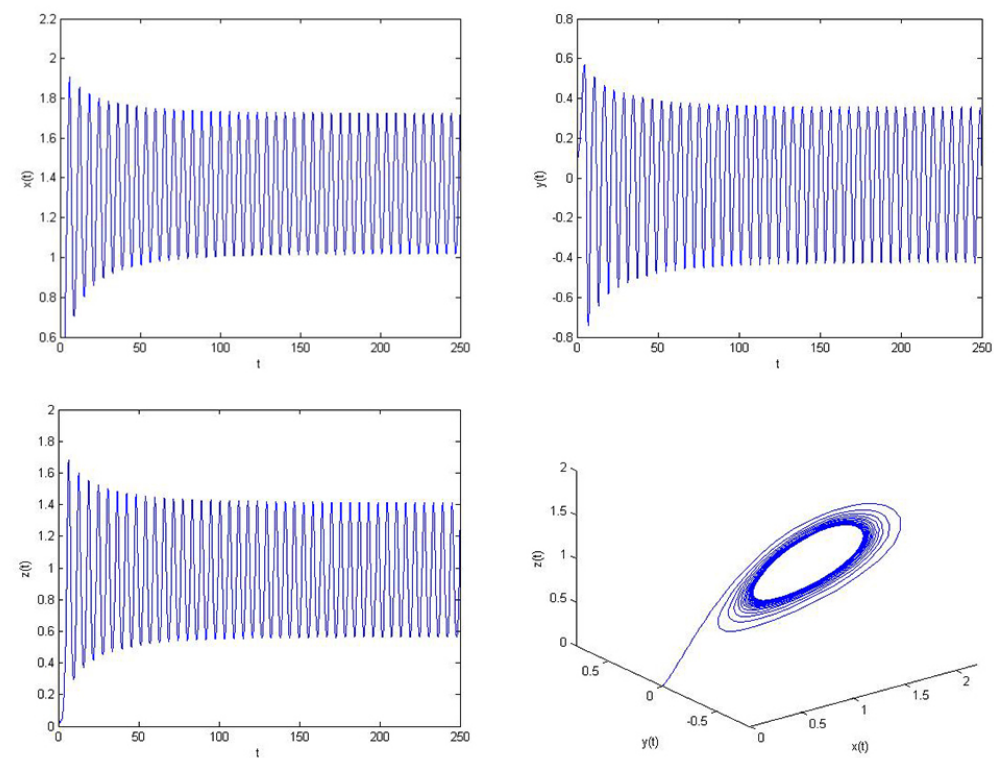

FIgURE 3. Bifurcating stable periodic solution for system (2.1) at $E_{+}=(1.414,0,1)$ with $\tau_{0}<\tau=0.2$.

stable periodic orbit. After $2 \pi$, that is at $\tau=\tau^{1}=5.6705$ (the position of (b) dashed vertical line), a Hopf bifurcation occurs and generates the unstable periodic orbit. At $\tau=\tau^{2}=11.1667$ (the position of (c) dashed vertical line), the Hopf bifurcation occurs and generated the unstable periodic orbit again. The second graph of Figure 4 is chosen the value of delay till 0.6 , which makes more clear to study the bifurcation diagram. In this paper, we study the Shimizu-Morioka system at Hopf bifurcation occurs and the stability of equilibrium. We also investigate the direction of the Hopf bifurcation and the stability of the bifurcating periodic solutions, by using the center manifold theory and normal form method. Our theoretical results and numerical simulations show that the chaos phenomena of system (1.1) can be controlled by delay. As the delay increases further, the numerical simulations show that the periodic solution disappears and the chaos attractor appears again. The obtained results can also be applied to the control and anticontrol of chaos phenomena of system (1.1). The other kinds of dynamical behaviors of system (4.1) out of the assumptions on the parameters have been disregarded. There are still abundant and complex dynamical behaviors and the topological structure of the new system should be completely and thoroughly investigated and exploited. We leave these as our future work.

\section{ACKNOWLEDGMENTS}

This research received a grant from China Scholarship Council (CSC). 

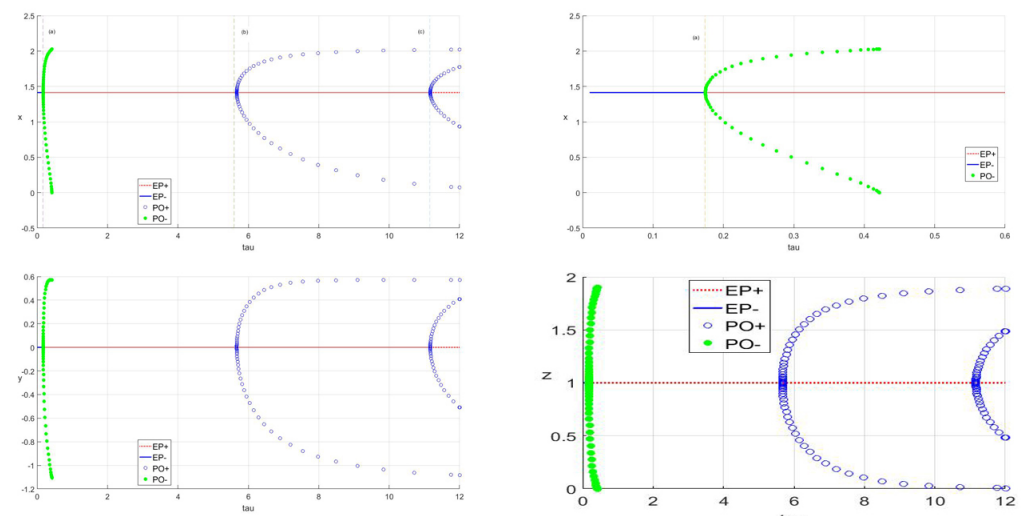

FIGURE 4. The bifurcation diagram for system (4.1). The blue solid line represents stable equilibrium point, and the red dotted line is unstable equilibrium point. Filled green circle represents stable periodic orbit, and open blue circles are unstable periodic orbits

\section{REFERENCES}

[1] L. J. Alvarez-Vázquez, F. J. Fernández, and R. Muñoz Sola, "Analysis of a multistate control problem related to food technology," J. Differential Equations, vol. 245, no. 1, pp. 130-153, 2008, doi: 10.1016/j.jde.2008.03.022. [Online]. Available: https://doi.org/10.1016/j.jde.2008.03.022

[2] K. Cooke, P. van den Driessche, and X. Zou, "Interaction of maturation delay and nonlinear birth in population and epidemic models," J. Math. Biol., vol. 39, no. 4, pp. 332-352, 1999, doi: 10.1007/s002850050194. [Online]. Available: https://doi.org/10.1007/s002850050194

[3] M. M. El-Dessoky, M. T. Yassen, and E. S. Aly, "Bifurcation analysis and chaos control in Shimizu-Morioka chaotic system with delayed feedback," Appl. Math. Comput., vol. 243, pp. 283-297, 2014, doi: 10.1016/j.amc.2014.05.072. [Online]. Available: https: //doi.org/10.1016/j.amc.2014.05.072

[4] K. Engelborghs, T. Luzyanina, and D. Roose, "Numerical bifurcation analysis of delay differential equations using DDE-BIFTOOL," ACM Trans. Math. Software, vol. 28, no. 1, pp. 1-21, 2002, doi: 10.1145/513001.513002. [Online]. Available: https://doi.org/10.1145/513001.513002

[5] J. Hale, Theory of functional differential equations, 2nd ed. Springer, 1977, applied Mathematical Sciences, Vol. 3 .

[6] B. D. Hassard, N. D. Kazarinoff, and Y. H. Wan, Theory and applications of Hopf bifurcation, ser. London Mathematical Society Lecture Note Series. Cambridge University Press, CambridgeNew York, 1981, vol. 41.

[7] D. C. Hill and D. S. Shafer, "Asymptotics and stability of the delayed Duffing equation," J. Differential Equations, vol. 265, no. 1, pp. 33-68, 2018, doi: 10.1016/j.jde.2018.02.029. [Online]. Available: https://doi.org/10.1016/j.jde.2018.02.029

[8] G.-P. Hu, W.-T. Li, and X.-P. Yan, "Hopf bifurcations in a predator-prey system with multiple delays," Chaos Solitons Fractals, vol. 42, no. 2, pp. 1273-1285, 2009, doi: 10.1016/j.chaos.2009.03.075. [Online]. Available: http://dx.doi.org/10.1016/j.chaos.2009.03.075

[9] X. Liao, F. Xu, P. Wang, and P. Yu, "Chaos control and synchronization for a special generalized Lorenz canonical system-the SM system," Chaos Solitons Fractals, 
vol. 39, no. 5, pp. 2491-2508, 2009, doi: 10.1016/j.chaos.2007.07.029. [Online]. Available: https://doi.org/10.1016/j.chaos.2007.07.029

[10] MATLAB, version 9.1.0 (R2016b). Natick, Massachusetts: The MathWorks Inc.

[11] A. V. Rezounenko and J. Wu, "A non-local PDE model for population dynamics with state-selective delay: local theory and global attractors," J. Comput. Appl. Math., vol. 190, no. 1-2, pp. 99-113, 2006, doi: 10.1016/j.cam.2005.01.047. [Online]. Available: https://doi.org/10.1016/j.cam.2005.01.047

[12] J. I. Richards and H. K. Youn, The theory of distributions: a nontechnical introduction. CUP Archive, 1995.

[13] L. F. Shampine and S. Thompson, "Solving DDEs in MATLAB," Appl. Numer. Math., vol. 37, no. 4, pp. 441-458, 2001, doi: 10.1016/S0168-9274(00)00055-6. [Online]. Available: https://doi.org/10.1016/S0168-9274(00)00055-6

[14] L. F. Shampine, S. Thompson, and J. Kierzenka, "Solving delay differential equations with dde23," URL http://www. runet. edu/ thompson/webddes/tutorial. pdf, 2000.

[15] A. L. Shilcprime nikov, "On bifurcations of the Lorenz attractor in the Shimizu-Morioka model," Phys. D, vol. 62, no. 1-4, pp. 338-346, 1993, homoclinic chaos (Brussels, 1991), doi: 10.1016/0167-2789(93)90292-9. [Online]. Available: https://doi.org/10.1016/0167-2789(93) 90292-9

[16] T. Shimizu and N. Morioka, "On the bifurcation of a symmetric limit cycle to an asymmetric one in a simple model," Phys. Lett. A, vol. 76, no. 3-4, pp. 201-204, 1980, doi: 10.1016/03759601(80)90466-1. [Online]. Available: https://doi.org/10.1016/0375-9601(80)90466-1

[17] H. Smith, "An introduction to delay differential equations with applications to the life sciences," vol. 57, pp. xii+172, 2011, doi: 10.1007/978-1-4419-7646-8. [Online]. Available: https://doi.org/10.1007/978-1-4419-7646-8

[18] G. Tigan and D. Turaev, "Analytical search for homoclinic bifurcations in the Shimizu-Morioka model," Phys. D, vol. 240, no. 12, pp. 985-989, 2011, doi: 10.1016/j.physd.2011.02.013. [Online]. Available: https://doi.org/10.1016/j.physd.2011.02.013

[19] S. Wiggins, Introduction to applied nonlinear dynamical systems and chaos. Springer Science \& Business Media, 2003, vol. 2.

[20] W. Zhang, J. Li, and M. Chen, "Global exponential stability and existence of periodic solutions for delayed reaction-diffusion BAM neural networks with Dirichlet boundary conditions," Bound. Value Probl., pp. 2013:105, 23, 2013, doi: 10.1186/1687-2770-2013-105. [Online]. Available: https://doi.org/10.1186/1687-2770-2013-105

[21] X. Zhang, Z. Zhang, and M. J. Wade, "Dynamical analysis of a competition and cooperation system with multiple delays," Bound. Value Probl., pp. Paper No. 111, 13, 2018, doi: 10.1186/s13661-018-1032-9. [Online]. Available: https://doi.org/10.1186/s13661-018-1032-9

\section{Author's address}

\section{Xin Zhang}

Nanjing University of Finance \& Economics, School of Applied Mathematics, 3 Wenyuan Ro., 210023 Nanjing, China

Jilin University, School of Mathematics, 2699 Qianjin St., 130012 Changchun, China

E-mail address: xinz15@mails.jlu.edu.cn 